\title{
DOMAIN STRUCTURE AND MAGNETIC PROPERTIES OF TERBIUM FERRITE-GARNET IN THE VICINITY OF THE MAGNETIC COMPENSATION POINT
}

\author{
Sharipov M.Z., Hayitov D.E., Rizoqulov M.N., Islomov U.N., Raupova I.B. \\ Bukhara Engineering - Technologies Institute, 200100, Bukhara, Uzbekistan, m.z.sharipov@rambler.ru \\ The domain structure of a thin single crystal plate of the iron garnet $\mathrm{Tb}_{3} \mathrm{Fe}_{5} \mathrm{O}_{12}$ has been \\ investigated using the magneto-optical method in the temperature range near the magnetic \\ compensation point of this ferrimagnet $T_{c}=248.6 \mathrm{~K}$. It has been shown that, when the temperature of \\ the sample approaches the magnetic compensation point, the domain width significantly increases, but \\ remains finite at $T=T_{c}$. The magnetic $H-T$ phase diagram determines the boundary between the multi \\ domain and domain free (uniformly magnetized) states of the sample. Here the magnetic $H-T$ phase \\ diagram has been constructed using the data on visual observations of the transformation of the domain \\ structure with variations in the temperature and external magnetic field.
}

Keywords: Ferrimagnetic, domain structure, magnetic compensation, phase diagram, domain boundary,

\section{Introduction}

Rare-earth ferrites-garnet (REFG), which include heavy RE ions, have a so-called magnetic compensation point - temperature $\mathrm{T}_{c}$ (below the Curie temperature), at which their spontaneous magnetic moment $M_{s}$ vanishes. From a practical point of view, REGF with sufficiently high $T_{c}$ are of interest as materials for the elemental base of magnetic microelectronics devices using the thermo-magnetic method of recording / erasing information [1, 2].

In this regard, direct visual observations of the domain structure (DS) of a thin single crystal $\mathrm{Tb}_{3} \mathrm{Fe}_{5} \mathrm{O}_{12}$ terbium garnet ferrite garnet in the temperature range [3, 4], including its magnetic compensation temperature $\left(\mathrm{T}_{\mathrm{c}}=248,6 \mathrm{~K}\right.$ [5]), were carried out, the results of which are presented below.

\section{Examples and experimental technique}

In our experiments, we used a $\mathrm{Tb}_{3} \mathrm{Fe}_{5} \mathrm{O}_{12}$ single crystal sample in the form of a plane-parallel plate with transverse dimensions $\approx 2 \times 3 \mathrm{~mm}^{2}$ and a thickness of $\approx 100 \mu \mathrm{m}$, oriented so that the [111] axis makes an angle of $\approx 10^{0}$ with the normal to its plane, and one of the axes has a smaller inclination to the plane of the sample compared with the other two (the error of the crystallographic orientation of the sample is $\sim \pm 2^{0}$ ) [6].

It is known $[7,8]$ that in REFG in a certain interval of the external magnetic field, the vectors $\mathbf{M}_{\mathrm{Fe}}$ and $\mathbf{M}_{\mathrm{R}}$ turn from antiparallel to parallel, i.e. a skewed magnetic phase occurs in which the angle between $\mathbf{M}_{\mathrm{Fe}}$ and $\mathbf{M}_{\mathrm{R}}$ is different from $180^{\circ}$. The critical field of the beginning of the turn of the sublattice magnetic moments

$$
H_{\kappa} \propto\left(M_{F e}-M_{R}\right) H_{0},
$$

where $\mathrm{H}_{0}$ is the exchange field acting between the iron and RE sublattices, whence it follows that near the magnetic compensation point $\mathrm{H}_{\mathrm{K}} \rightarrow 0$, i.e. at $\mathrm{T} \rightarrow \mathrm{T}_{\mathrm{c}}$, the scattering fields created by neighboring domains may be sufficient to start the reversal of the vectors $\mathbf{M}_{\mathrm{Fe}}$ and $\mathbf{M}_{\mathrm{R}}$. Therefore, in the general case, with normal incidence of light on the plane of the sample, the spontaneous Faraday effect in REFG can be represented as: 


$$
\theta_{F}=\left(a \vec{M}_{F e} \cos \theta_{1}+b M_{R} \cos \theta_{2}\right)
$$

where $a$ and $b$ - are magneto-optical coefficients depending on the frequency of light; $\theta_{1}, \theta_{2}$ are the angles formed by the vectors $\mathbf{M}_{\mathrm{Fe}}$ and $\mathbf{M}_{\mathrm{R}}$ with a normal to the plane of the sample (in the collinear magnetic phase of the crystal $\left.\theta_{1}=\theta_{2}\right) ; l$ - is the thickness of the sample.

In $\mathrm{Tb}_{3} \mathrm{Fe}_{5} \mathrm{O}_{12}$ at $T<T_{\mathrm{c}} \mathbf{M}_{\mathrm{R}}>\mathbf{M}_{\mathrm{Fe}}$, therefore, the vector $\mathbf{M}_{\mathrm{R}}$ is oriented in the direction of the external magnetic field. At $T>T_{\mathrm{c}}$, the situation is the opposite - the vector $\mathbf{M}_{\mathrm{Fe}}$ is directed toward H. Thus, at the magnetic compensation point, the vectors $\mathbf{M}_{\mathrm{Fe}}$ and $\mathbf{M}_{\mathrm{R}}$ are rotated by $180^{\circ}$, which, in accordance with (1), leads to a change in the sign of the Faraday rotation angle, which can be determined by a sharp change in the color of the observed domains. Moreover, as can be seen from (1), even at $\left(\mathbf{M}_{\mathrm{Fe}}+\mathbf{M}_{\mathrm{R}}\right)=0$ (at $T=T_{\mathrm{c}}$ ), the Faraday Effect does not vanish, which allows us to observe the DS of the sample when the temperature passes through the point of magnetic compensation.

\section{Experimental results and discussion}

As observations have shown, in accordance with expectations in the entire studied temperature range of 85-295 $\mathrm{K}$ at $H=0$, the DS of the sample formed domains in the form of light (visually red) and dark bands of approximately equal width with clearly defined boundaries [9]. In this case, an external magnetic field parallel to the middle direction of the domain walls strongly affected the domain width, transferring the sample at a certain $T$-dependent value of $H$ to a homogeneous (single-domain) state, while the field oriented in the plane of the sample is perpendicular to the domain wall (DW), practically did not change the width of the domains up to the maximum intensity $H=60$ Oe used in the experiment. Such a behavior of the domain structure obviously means that the vectors $\mathbf{M}_{\mathrm{s}}$ in neighboring domains are antiparallel to each other and oriented along domain boundaries (i.e., $180^{\circ}$ - DS is realized in the sample).

As an example in Fig.1 shows a number of photographs taken during the heating of the sample from $T=85 K$ (heating rate $\sim 0.2 \mathrm{~K} / \mathrm{s}$ ), illustrating the general nature of the change in its DS depending on temperature. A series of photographs a $\div$ e was obtained immediately after cooling the sample to $T=85 K$; each photograph of the series shown in fig. 2 - after demagnetization of the sample at a given $T$ in an alternating magnetic field (changing with a frequency of $50 \mathrm{~Hz}$ ), the amplitude of which decreased from the maximum value $H=60$ Oe to zero.
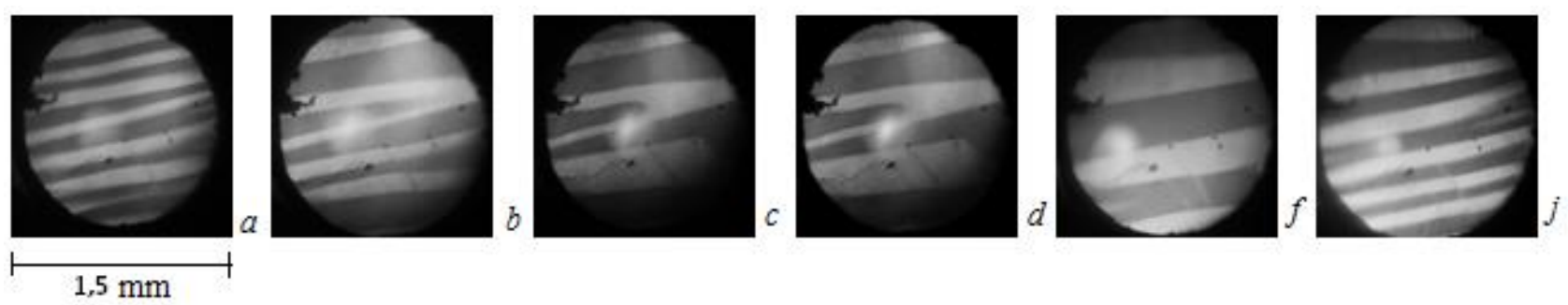

Fig. 1.Images of the domain structure of the sample obtained at different temperatures during heating: $205(a), 237(b), 248(c), 251(d), 256(f)$ and $295 \mathrm{~K}(j)$.
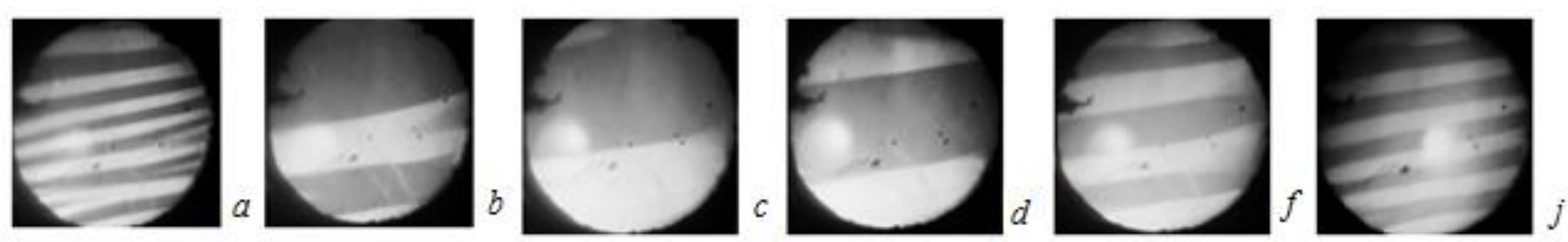

Fig. 2. Images of the "equilibrium" domain structure of the sample obtained at different temperatures during heating: $205(a), 236(b), 248(c), 251(d), 260(f)$ and $295 \mathrm{~K}(j)$. At each temperature, the sample was demagnetized in an alternating magnetic field with amplitude decreasing to zero. 
It was assumed that the demagnetization procedure allows one to obtain a domain configuration close to equilibrium at a given temperature. As a rule, after this procedure, for each value of $T, 2-3$ domain configurations were observed, slightly differing by the position of the domain walls, however, the average width of the domains arising from case to case remained almost constant. The temperature dependence of the average domain width of the "equilibrium" DS sample is shown in Fig. 3. Note that, when approaching the point of magnetic compensation, a deterioration in the magneto-optical contrast and a change in the color shades of images of neighboring domains were observed.

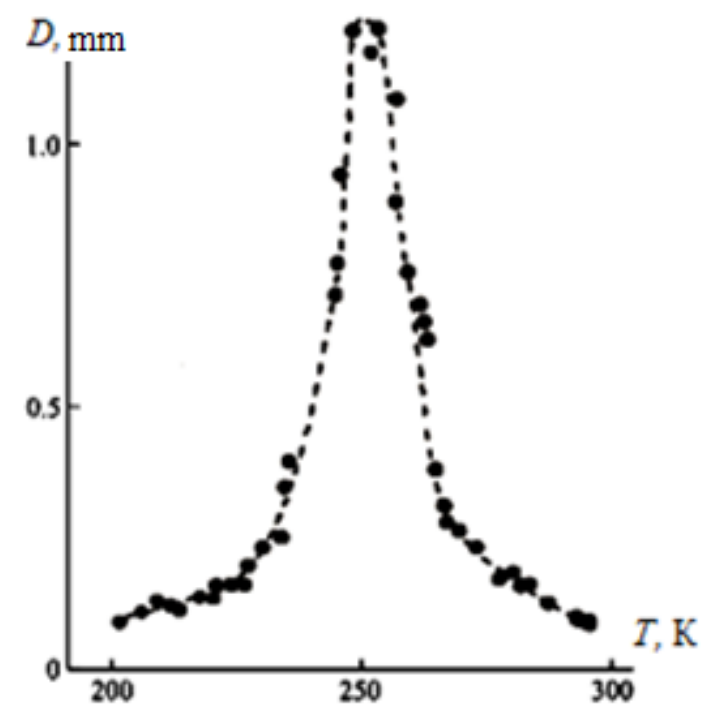

Fig. 3. Temperature dependence of the average domain width of the "equilibrium" domain structure of the sample.

The single domain state of the sample could be observed under the action of an external field $\mathbf{H}$, which has a noticeable projection onto the direction of the domain walls. So, in a field oriented along the direction of domain walls at an angle of $\approx 10^{\circ}$ to the plane of the sample (along the assumed direction of the distinguished light axis), the single-domain state of the sample was observed near $T_{\mathrm{c}}$ at $H>4$ Oe. In Fig. 4 shows photographs demonstrating the behavior of the DS of a sample with a change in temperature at the above orientation of the magnetizing field at $H=5$ Oe, from which it can be seen that the single domain state of the sample occurs in a certain temperature range to the right and left of $T_{\mathrm{c}}$. As observations showed, with an increase in $H$, these temperature ranges of the "single domain" gradually expanded, until at $H \approx 7$ Oe they merged into one general one. In the "single domain" region, the image of the sample has a uniform color over the entire area; when the temperature passes through the compensation point, the color of the image of the sample (in accordance with (1)) changes abruptly.
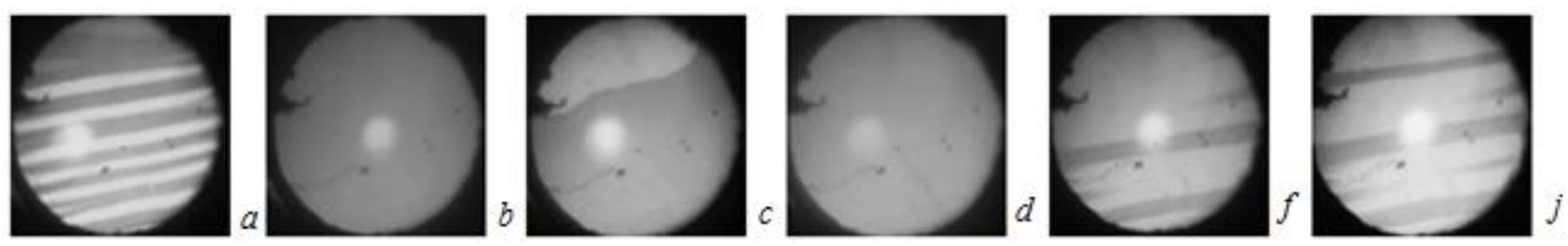

Fig. 4. Images of the domain structure of the sample obtained at different temperatures during heating in a magnetic field $H=4,50 e: 200(a), 240(b), 248(c), 270(d), 260(f)$, and $295 \mathrm{~K}(j)$. At each temperature, the sample was demagnetized in an alternating magnetic field with amplitude decreasing to zero. Vector $\mathbf{H}$ is parallel to O.L.N. 
In fig. 5 shows the experimentally obtained magnetic $H-T$ diagram that defines the boundary between multi-domain and single-domain (uniformly magnetized) states of a sample. Each point of the diagram was determined by the results of visual observations of the disappearance of domain walls in the image of the sample with a change in $\mathrm{H}$ and $\mathrm{T}$ (in the experiment, the sample was first cooled to $T=85 \mathrm{~K}$, then the magnetic field of the specified strength was turned on and the process of changing the DS during heating of the sample was observed, while at each fixed temperature, the sample was subjected to a demagnetization procedure).

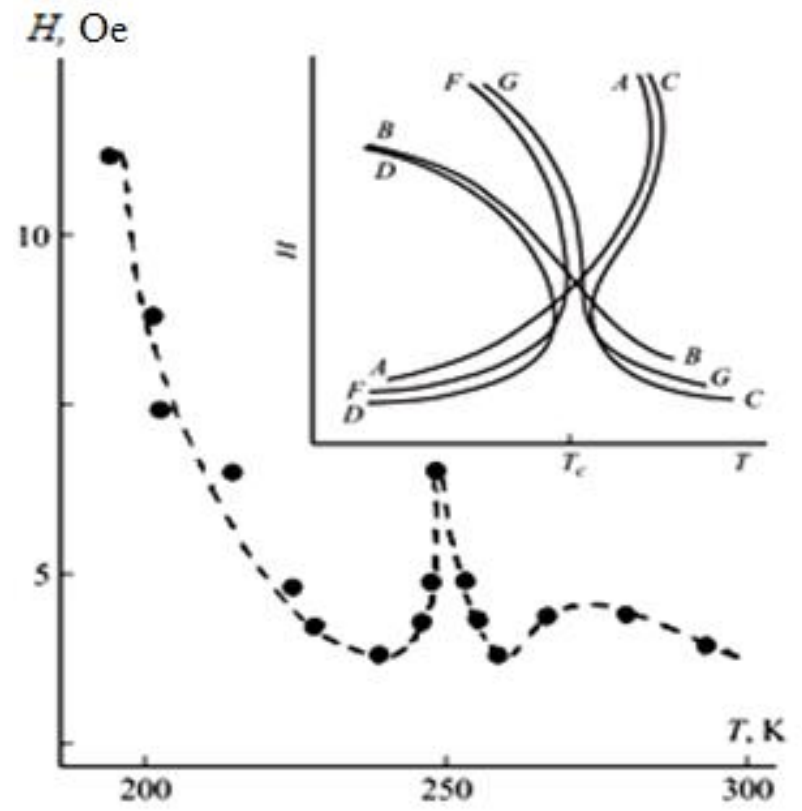

Fig. 5.Temperature dependence of the magnetic field at which the sample goes into a single domain state. The direction of the vector $\mathbf{H}$ is parallel to O.L.N. - crystallographic direction $\langle 111\rangle$. Inset: phase $H-$ $T$ diagram describing the magnetic state of the rare-earth phase group at $\mathbf{H}\|\|$ [111] [7], AA and BB are the lines of phase stability loss $(\varphi=0)$ and $(\varphi=\pi)$, SS and FF are the boundaries regions of stability of states $(0$ $<\varphi<\pi / 2, \xi=\pi / 2,7 \pi / 6,11 \pi / 6)$, DD and GG are the boundaries of the region of stability of states $(\pi / 2$ $<\varphi<\pi, \xi=\pi / 6,5 \pi / 6,3 \pi / 2)$, where $\varphi$ and $\xi$ are respectively the polar and azimuthal angles of the vector

$\mathbf{M}_{\mathrm{Fe}}$ in the Cartesian coordinate system \{[110], [112], [111]\} (the [111] axis is the polar axis).

The $H-T$ diagram of the sample obtained in this way is in good agreement (on a qualitative level) with the magnetic phase diagram describing the magnetic state of the REFG in $\mathrm{H} \|\langle 111\rangle$ calculated in [8] (see inset of Fig. 5). From the fragment shown in the inset Fig. 5 of the phase diagram of the REFG, it can be seen that at $T \rightarrow T_{\mathrm{c}}$, the angular phases of the crystal appear earlier than the magnetic moments of the iron and rare-earth sublattices are equal in magnitude. The stability loss lines of various magnetic phases of a crystal determine the boundaries of the existence of domains with the corresponding directions of magnetic moments (DS arises due to demagnetizing fields). This suggests that the experimentally observed decrease in the contrast of the image of the domains and a change in their color in the immediate vicinity of the sample temperature to $T_{\mathrm{c}}$ is associated with the transition of the crystal from the collinear magnetic phase to the angular one. In this case, the skew of the vectors $\mathbf{M}_{\mathrm{Fe}}$ and $\mathbf{M}_{\mathrm{R}}$ will lead to a decrease in their projection on the direction of the light propagating in the crystal, and as a result to a decrease in the magnitude of the Faraday effect (see (1)).

From fig. 1 and fig. 2, it follows that although the width of the domains at $T \rightarrow T_{\mathrm{c}}$ increases significantly, nevertheless, in contrast to the results of a theoretical analysis of the DS of a planeparallel ferrimagnet plate obtained in $[10,11]$, the domains in the sample do not disappear in the entire temperature range studied, i.e. e. at $H=0$, the sample does not transform into a uniformly magnetized state. In addition, it follows from the theory developed in $[9,10]$ that, at $T \rightarrow T_{\mathrm{c}}$, the 
maximum domain width reaches a value comparable to the plate thickness, whereas from Fig. 1 and fig. 2 shows that in our case the maximum domain width is more than 10 times the thickness of the sample.

\section{Conclusion}

Thus, the performed studies have shown that, in a plane-parallel plate of the $\mathrm{Tb}_{3} \mathrm{Fe}_{5} \mathrm{O}_{12}$ garnet ferrite garnet, at $\mathrm{H}=0$, when the temperature passes through the point of magnetic compensation, the DW does not disappear. The explanation of this fact was based on the magnetic phase diagram of the REFG obtained in [8]. However, there are other possible reasons for the existence of domains in the sample near $T_{c}$ - for example, defects in the crystal lattice or the presence of a noticeable entropy contribution to the thermodynamic potential of a crystal, which makes its multi-domain state energetically more favorable than a single-domain state (as is the case in collinear antiferromagnets) [12]. An unambiguous choice of the reason leading to the existence of an REFG $\mathrm{DS}$ at $\mathrm{T} \rightarrow \mathrm{T}_{\mathrm{c}}$ is possible, in our opinion, if we trace the dynamics of the spatial orientation of the sublattice magnetic moments of the crystal. In particular, additional information can be obtained from a detailed analysis of the color change of domains when the temperature changes, made using a computer-based method for processing optical images.

\section{REFERENCES}

1 Rainer Waser. Nanoelectronics and information technology. Advanced electronic materials and novel devices. Wiley-VCH GmbH \& Co. KGaA, 2003, 1001 p.

2 Shavrov V.G., Sheglov V.I. Dinamika namagnichennosti $v$ usloviyh izmeneniya orientachii. Moscow, Fizmatlit, 2019, 468p. [in Russian].

3 Boidedaev S.R., Dzhuraev D.R., Sokolov B.Yu., Sharipov M.Z. Magnetooptical method of investigation of the magnetic inhomogeneity of easy-plane antiferromagnets with a weak ferro-magnetism. Optics and Spectroscopy, 2008, Vol. 104, No. 4, pp. 604-609.

4 Sharipov M.Z., Mirzhonova N.N., Hayitov D.E. Effect of inhomogeneous radially directed mechanical stresses on the domain structure of a $\mathrm{FeBO}_{3}$ single crystal. Eurasian Physical Technical Journal, 2019, 16(1), pp. 35-41.

5 Grigoryeva I.S., Meilikhova E.Z. Physical quantities. Moscow, Energoatomizdat, 1991, 223 p. [in Russian].

6 Sokolov, B.Y., Sharipov, M.Z. Magneto-optical properties of the iron garnet $\mathrm{Tb}_{3} \mathrm{Fe}_{5} \mathrm{O}_{12}$ near the magnetic compensation temperature. Physics of the Solid State, 2014, Vol. 56, No. 5, pp. 975 - 979.

7 Bukharaev A.A., Zvezdin A.K., Pyatakov A.P., Fetisov Yu.K. Straintronics: a new trend in microand nanoelectronics and materials science. Phys. Usp., 2018, Vol. 61, Issue 12, pp. 1175 - 1212.

8 Pyatakov A.P., Zvezdin A.K. Magnetoelectric and multiferroic media. Phys. Usp., 2012, Vol. 55, Issue 6, pp. $557-581$.

9 Sokolov B.Yu., Talabov M.D., Sharipov M.Z. Domain structure of a thin single-crystal plate of terbium ferrite garnet near the point of magnetic compensation. Physics of the Solid State. 2013, Vol. 55, No.2, pp. $278-283$.

10 Bogdanov A.N., Yablonsky D.A. On the theory of the domain structure of ferrimagnets. FTT. 1980, Vol. 22, No. 3, pp.680 - 687. [in Russian].

11 Baryakhtar V.G., Bogdanov A.N., Yablonsky D.A. The domain structure of ferrites in the vicinity of the compensation temperature. FTT. 1986, Vol. 28, No. 1, pp. 87 - 94. [in Russian]

12 Shavrov V.G., Sheglov V.I. Ferromagnitniy rezonans $v$ usloviyah orientatsionnogo perehoda. Moscow, Fizmatlit, 2018, 468 p. [in Russian]. 